Pacific Journal of Mathematic 


\title{
CHAIN CONDITIONS IN FREE PRODUCTS OF LATTICES WITH INFINITARY OPERATIONS
}

\author{
G. Grätzer, A. Hajnal and David Kelly
}

There are many facts known about the size of subsets of certain kinds in free lattices and free products of lattices. Examples: every chain in a free lattice is at most countable; every "large" subset contains an independent set; if the free product of a set of lattices contains a "long" chain, so does the free product of a finite subset of this set of lattices. Here we investigate these problems in the setting of a variety $V$ of m-lattices, where $\mathfrak{m}$ is an infinite regular cardinal. An m-lattice $L$ is a lattice in which for any nonempty set $S$ with $|S|<\mathfrak{m}$, the meet and join exist in $L$. We obtain generalizations of many finitary results to the m-complete case. Our basic set-theoretic tool is the ErdösRado theorem.

1. Preliminaries. Lower-case German letters denote cardinals. Lower-case Greek letters denote ordinals; cardinals are identified with initial ordinals.

A family $\left(S_{i} \mid i \in I\right)$ of sets is a $\Delta$-system with kernel $D$ iff $S_{i} \cap S_{j}=D$ whenever $i \neq j$ and $i, j \in I$. The cardinal $\mathfrak{n}$ is strongly $\mathfrak{m}$-inaccessible iff $\mathfrak{b}^{a}<\mathfrak{n}$ whenever $\mathfrak{a}<\mathfrak{m}$ and $\mathfrak{b}<\mathfrak{n}$. For example, $\left(2^{\mathfrak{m}}\right)^{+}$is strongly $\mathfrak{m}$-inaccessible [2, Lemma 1.26], where $2^{\mathrm{m}}=\Sigma\left(2^{\mathfrak{a}} \mid \mathfrak{a}<\right.$ $\mathfrak{m})$. Note that $2^{\mathfrak{m}} \geqq \mathfrak{m}$, and equality holds if the Generalized Continuum Hypothesis (G. C. H) is assumed. Under G. C. H., if $n>m$ is the successor of a regular cardinal, then it is strongly $\mathrm{m}$-inaccessible.

Let $\mathfrak{n}>\mathfrak{m}$ be regular and strongly $\mathfrak{m}$-inaccessible. The ErdösRado theorem [3, Lemma 1] states that for any family $\left(S_{\alpha} \mid \alpha<\mathfrak{n}\right)$ of sets with $\left|S_{\alpha}\right|<\mathfrak{m}$ whenever $\alpha<\mathfrak{n}$, there is $N \leqq \mathfrak{n}$ with $|N|=\mathfrak{n}$ such that $\left(S_{\alpha} \mid \alpha \in N\right)$ is a $\Delta$-system.

In this paper, $\mathfrak{m}$ is an infinite regular cardinal. The prefix " $m$-" is consistently used to extend concepts from the usual case of finitary joins and meets; for further details, see [6] and [7].

A variety $V$ of m-lattices or m-variety is a class of $m$-lattices that is closed under $\mathfrak{m}$-homomorphic images, m-sublattices and products. $\quad V$ shall always denote a nontrivial m-variety.

The $V$-free m-product $L$ of a family $\left(L_{i} \mid i \in I\right)$ of m-lattices in $V$ is the m-lattice $L \in V$ (unique up to isomorphism) that contains each $L_{i}(i \in I)$ as an m-sublattice and is m-generated by $X=\cup\left(L_{i} \mid i \epsilon\right.$ I) (disjoint union) such that any family $\varphi_{i}: L_{i} \rightarrow K$ of m-homomorphisms into any $K \in V$ can be extended to an m-homomorphism 
of $L$ into $K$. In particular, if each $L_{i}(i \in I)$ is a one-element lattice, then $L$ is the $V$-free $m$-lattice generated by $X$. We omit mention of $V$ if it is the variety $\boldsymbol{L}_{\mathrm{u}}$ of all $\mathfrak{m}$-lattices. We also omit $\mathfrak{m}$ if $\mathfrak{m}=\aleph_{0}$.

Let $\boldsymbol{X}=\left\{\boldsymbol{x}_{\alpha} \mid \alpha<\mathfrak{m}\right\}$ be a set of variables. The $\mathfrak{m}$-polynomials in $X$, defined in [6], are built up using formal joins and meets of less than $\mathfrak{m}$ elements, starting from $\boldsymbol{X}$. The set $\boldsymbol{P}_{\mathrm{m}}(\boldsymbol{X})$ of all $\mathfrak{m}-$ polynomials in $X$ has cardinality $2^{\mathrm{m}}$. Let $L$ be an m-lattice that is m-generated by a set $X$. We can express any element $a \in L$ as $a=\boldsymbol{p}(\bar{a})$ where $\boldsymbol{p} \in \boldsymbol{P}_{\mathrm{m}}(\boldsymbol{X}), \boldsymbol{Y} \subset \boldsymbol{X}$ is the set of variables appearing in $p$, and $\bar{a}$ is a mapping from $Y$ to $X$. By induction on the rank of $p$ (see [6]), it is easily shown that any $a \in L$ has such a representation with $\bar{a}$ one-to-one (that is, distinct variables are substituted by distinct elements of $X$ ); such a representation is called proper. A subset $Y$ of an m-lattice is m-irredundant iff the following condition and its dual hold: whenever $a \leqq \mathrm{~V} B$ with $a \in Y, B \subseteq Y$ and $0<|B|<\mathfrak{m}$, it follows that $a \in B$. In particular, an $\mathfrak{m}$-irredundant subset is an antichain.

2. The results. In a $V$-free lattice, every chain is countable. This result is proved in F. Galvin and B. Jónsson [4] in a much sharper form. Our first result generalizes their sharper form.

THEOREM 1. Let $V$ be a nontrivial $\mathfrak{m}$-variety, and let $\mathfrak{n}$ be a regular cardinal that is greater than $\mathfrak{m}$ and strongly $\mathfrak{m}$-inaccessible. If a set of cardinality $\mathfrak{n}$ is a subset of a $V$-free $\mathfrak{m}$-lattice, then it contains an $\mathrm{m}$-irredundant subset of the same cardinality.

CoRollary 1. Every $V$-free $\mathfrak{m}$-lattice satisfies the $\left(2^{m}\right)^{+}$-chain condition, that is, it has no chain of cardinality $\left(2^{\mathrm{m}}\right)^{+}$.

A subset $S$ of a lattice is quasidisjoint iff $a \wedge b=c \wedge d$ whenever $a, b, c, d \in S$ with $a \neq b$ and $c \neq d$. A lattice satisfies the n-quasidisjointness condition iff it contains no quasidisjoint set of cardinality $\mathfrak{n}$. Since no $\mathfrak{m}$-irredundant set with more than two elements can be quasidisjoint, we have

CoROLlaRY 2. Every $V$-free $\mathfrak{m}$-lattice satisfies the $\left(2^{\mathfrak{m}}\right)^{+}-q u a s i$ disjointness condition.

A subset $Y$ of a free m-lattice $L$ is $\mathfrak{m}$-independent iff the m-sublattice of $L$ m-generated by $Y$ is (isomorphic to) the free m-lattice generated by $Y$. Sinde m-irredundancy is equivalent to $\mathfrak{m}$-independency for subsets of a free m-lattice [6], we obtain a 
result due to F. Galvin and B. Jónsson [4] in the $\mathfrak{m}=\boldsymbol{\aleph}_{0}$ case.

COROLLARY 3. Let $\mathfrak{n}$ be a regular cardinal that is greater than $\mathfrak{m}$ and strongly $\mathfrak{m}$-inaccessible. If a set of cardinality $\mathfrak{n}$ is a subset of a free $\mathfrak{m}$-lattice, then it contains an $\mathfrak{m}$-independent subset of the same cardinality.

B. Jónsson [9] proved that the $V$-free product of lattices $\left(L_{i} \mid i \epsilon\right.$ $I$ ) satisfies the $\mathfrak{m}$-chain condition ( $\mathfrak{m}$ is regular and $>\boldsymbol{\aleph}_{0}$ ) iff for all finite $I^{\prime} \subseteq I$, the $V$-free product of $\left(L_{i} \mid i \in I^{\prime}\right)$ satisfies the $\mathfrak{m}$-chain condition. Our next result generalizes this.

THEOREM 2. Let $V$ be an $\mathfrak{m}$-variety. Let $\mathfrak{n}$ be a regular cardinal that is greater than $\mathfrak{n}$ and strongly $\mathfrak{m}$-inaccessible. Let $L$ be the $V$-free $\mathfrak{m}$-product of the $\mathfrak{m}$-lattices $L_{i} \in V, i \in I$. If, for all $J \subseteq I$ with $|J|<\mathfrak{m}$, the free $\mathfrak{m}$-product of $\left(L_{\imath} \mid i \in J\right)$ satisfies the $\mathfrak{n}$-chain condition, then so does $L$.

If $\mathfrak{n}$ is singular and cofinal with $\aleph_{0}$, then there are two lattices satisfying the $\mathfrak{n}$-chain condition whose $V$-free product does not satisfy the $\mathfrak{n}$-chain condition. If $\mathfrak{n}$ is cofinal with $\aleph_{0}$, then there are countably many chains of cardinality $<\mathfrak{n}$, whose $V$-free product does not satisfy the $\mathfrak{n}$-chain condition (B. Jónsson [9] and G. Grätzer and $H$. Lakser [8]). The next two results are the analogues for m-lattices.

$\boldsymbol{D}_{m}$ will denote the smallest nontrivial variety of $\mathfrak{u t}$-lattices (generated by 2 , the two-element m-lattice).

THEOREM 3. Let $\mathfrak{n}$ be a strongly $\mathfrak{m}$-inaccessible singular cardinal whose cofinality is greater than $2^{\mathfrak{m}}$. Then there are two Boolean $\mathfrak{m}$-algebras in $\boldsymbol{D}_{\mathfrak{m}}$ satisfying the $\mathfrak{n}$-chain condition such that their $V$-free $\mathfrak{m}$-product does not satisfy the $\mathfrak{n}$-chain condition.

THEOREM 4. If $\mathfrak{n}>\mathfrak{m}$ is an infinite cardinal of cofinality $\mathfrak{m}_{0}$ with $\mathfrak{m}_{0} \leqq \mathfrak{m}$, then there are $\mathfrak{m}_{0}$ complete chains of cardinality less than $\mathfrak{n}$ whose $V$-free $\mathfrak{m}$-product does not satisfy the $\mathfrak{n}$-chain condition.

Some open problems are listed in $\S 6$.

3. Proof of Theorem 1. Let $\mathfrak{n}$ be as in the statement of the theorem, let $L$ be the $V$-free m-lattice generated by a set $X$, and let $Y$ be a subset of $L$ with $|Y|=\mathfrak{n}$. Since $\mathfrak{n}$ is regular, $2^{\mathrm{m}}<\mathfrak{n}$. 
Hence, we can assume that each element of $Y$ has a proper representation $a=p(\bar{a})$, where the same m-polynomial $\boldsymbol{p}$ is used for each element of $Y$. For notational simplicity, we further assume that, for some cardinal $\mathfrak{m}_{0}<\mathfrak{m}, \bar{a}=\left\langle x_{\alpha}^{a} \mid \alpha<\mathfrak{m}_{0}\right\rangle$ whenever $a \in Y$, where $x_{\alpha}^{a} \in X$ for all $\alpha<\mathfrak{m}_{0}$. (Note that $x_{\alpha}^{a} \neq x_{\beta}^{a}$ for $\alpha \neq \beta$.)

Consider the sets $S_{a}=\left\{x_{\alpha}^{a} \mid \alpha<\mathfrak{m}_{0}\right\}$ for $a \in Y$. By the Erdös-Rado theorem, there is a subset $Y^{\prime} \cong Y$ with $\left|Y^{\prime}\right|=\mathfrak{n}$ such that $\left(S_{a} \mid a \in\right.$ $\left.Y^{\prime}\right)$ is a $\Delta$-system, whose kernel we denote by $D$. For each $a \in Y^{\prime}$, the inclusion $D \subseteq S_{a}$ induces a map $\psi_{a}: D \rightarrow \mathfrak{m}_{0}$ in the obvious way. Since $\left|\left\{\psi_{a} \mid a \in Y^{\prime}\right\}\right| \leqq \mathfrak{m}_{0}^{\mathfrak{m}_{0}}=2^{\mathfrak{m}_{0}}<\mathfrak{n}$, we can assume that $\psi_{a}$ is the same map for all $a \in Y^{\prime}$. This means that if $x_{\alpha}^{a} \in D\left(a \in Y^{\prime}, \alpha<\mathfrak{m}_{0}\right)$, then $x_{\alpha}^{a}=x_{\beta}^{b}$ for all $b \in Y^{\prime}$.

We first show that $Y^{\prime}$ is an antichain in $L$. Supposing otherwise, there are $a, b \in Y^{\prime}$ with $a<b$. We define an m-homomorphism $\varphi: L \rightarrow L$ as follows: $\varphi\left(x_{\alpha}^{a}\right)=x_{\alpha}^{b}$ and $\varphi\left(x_{\alpha}^{b}\right)=x_{\alpha}^{a}$ whenever $\alpha<\mathfrak{m}_{0}$; otherwise, if $x \in X, \varphi(x)=x$. Then, $\varphi(a)=b$ and $\varphi(b)=a$. Applying $\varphi$ to the inequality $a<b$, we conclude that $b \leqq a$, a contradiction.

Let $a \leqq \bigvee B$ with $a \in Y^{\prime}, B \cong Y^{\prime}$ and $0<|B|<\mathfrak{m}$. Suppose that $a \notin B$. Fix $c \in B$. We define an m-homomorphism $\varphi: L \rightarrow L$ as follows: $\psi\left(x_{\alpha}^{b}\right)=x_{\alpha}^{c}$ whenever $b \in B$ and $\alpha<\mathfrak{m}_{0}$; otherwise, if $x \in X$, $\varphi(x)=x$. Then $\varphi(a)=a$ and $\varphi(b)=c$ whenever $b \in B$.

Applying $\varphi$ to the inequality $a \leqq \bigvee B$, we conclude that $a<c$, contradicting that $Y^{\prime}$ is an antichain. This completes the proof of the theorem.

4. Proof of Theorem 2. We prepare the proof of Theorem 2 by

Lemma 1 . Let $L$ be the $V$-free $\mathfrak{m}$-product of $\mathfrak{m}$-lattices $L_{0}, L_{1}, L_{2}$; let $L_{3}$ be an $\mathfrak{m}$-lattice and let $e \in L_{3}$; and let $\boldsymbol{p}=\boldsymbol{p}(\boldsymbol{x}, \boldsymbol{y})$ and $\boldsymbol{q}=$ $\boldsymbol{q}(\boldsymbol{x}, \boldsymbol{y})$ be m-polynomials whose variables are $\boldsymbol{x}=\left\langle\boldsymbol{x}_{\alpha} \mid \alpha<\beta\right\rangle$ and $\boldsymbol{y}=\left\langle\boldsymbol{y}_{\alpha}\right| \alpha\langle\gamma\rangle$. Let $\boldsymbol{a}$ and $\boldsymbol{b}$ be $\beta$-sequences of elements of $L_{0}$; let $c$ and $\boldsymbol{d}$ be $\gamma$-sequences of elements of $L_{1}$ and $L_{2}$ respectively, and let $\boldsymbol{e}$ be the $\gamma$-sequence with constant entry $e$. If

$$
p(a, c) \leqq q(b, d)
$$

in $L$ and

$$
p(\boldsymbol{a}, e)=q(b, e)
$$

in the $V$-free product $K$ of $L_{0}$ and $L_{3}$, then

$$
p(\boldsymbol{a}, \boldsymbol{c})=\boldsymbol{q}(\boldsymbol{b}, \boldsymbol{d})
$$


in $L$.

Proof. Let $L^{b}=L \cup\{0,1\}$, the m-lattice formed by adding a new zero and one to $L$. It is easily seen that $L^{b} \in V$. Further, let 0 and 1 be the $\gamma$-sequences with constant entry 0 and 1 , respectively. We are assuming that (i) $\boldsymbol{p}(\boldsymbol{a}, \boldsymbol{c}) \leqq \boldsymbol{q}(\boldsymbol{b}, \boldsymbol{d})$ in $L$ and (ii) $\boldsymbol{p}(\boldsymbol{a}, \boldsymbol{e})=\boldsymbol{q}(\boldsymbol{b}, \boldsymbol{e})$ in $K$. By considering the $\mathfrak{m}$-homomorphism from $L$ to $L^{b}$ that maps $L_{0}$ identically, everything in $L_{1}$ to 1 , and everying in $L_{2}$ to 0 , we conclude from (i) that $\boldsymbol{p}(\boldsymbol{a}, \mathbf{1}) \leqq \boldsymbol{q}(\boldsymbol{b}, 0)$ in $L^{b}$. Using (ii) and the obvious $\mathfrak{m}$-homomorphisms from $K$ to $L^{b}$, we also conclude that $\boldsymbol{p}(\boldsymbol{a}, \mathbf{0})=\boldsymbol{q}(\boldsymbol{b}, \mathbf{0})$ and $\boldsymbol{p}(\boldsymbol{a}, \mathbf{1})=\boldsymbol{q}(\boldsymbol{b}, \mathbf{1})$ in $L^{b}$. Thus, $\boldsymbol{q}(\boldsymbol{b}, \mathbf{1}) \leqq \boldsymbol{p}(\boldsymbol{a}, \mathbf{0})$ in $L^{b}$. It is easily shown by induction on the rank that $\boldsymbol{p}(\boldsymbol{a}, 0) \geqq \boldsymbol{p}(\boldsymbol{a}, \boldsymbol{c})$ and $\boldsymbol{q}(\boldsymbol{b}, \boldsymbol{d}) \leqq \boldsymbol{q}(\boldsymbol{b}, \mathbf{1})$ in $L^{b}$. Therefore, $\boldsymbol{q}(\boldsymbol{b}, \boldsymbol{d}) \geqq \boldsymbol{p}(\boldsymbol{a}, \boldsymbol{c})$ in $L$, the desired conclusion.

Let $\mathfrak{n}$ be as in the statement of Theorem 2 , let $L$ be the $V$-free m-product of the family $\left(L_{i} \mid i \in I\right)$ of m-lattices, and let $X=\mathrm{U}\left(L_{i} \mid i \in\right.$ $I$ ), a subset of $L$. Suppose that $C$ is a chain in $L$ of cardinality $\mathfrak{n}$. As in the proof of Theorem 1, we can assume that there is a single $\mathfrak{m}$-polynomial $\boldsymbol{p}$ and a cardinal $\mathfrak{m}_{0}<\mathfrak{m}$ such that each element $a$ of $C$ has a representation $a=p\left(\left\langle x_{\alpha}^{a} \mid \alpha<\mathfrak{m}_{0}\right\rangle\right)$, where $x_{\alpha}^{a} \in X$ for all $\alpha<\mathfrak{m}_{0}$. For $x \in X, i(x)$ denotes the element $j$ of $I$ such that $x \in L_{j}$. Since there are less than $\mathfrak{n}$ equivalence relations on $\mathfrak{m}_{0}$, we can further assume that, whenever $\alpha, \beta<\mathfrak{m}_{0}$, if the equality $i\left(x_{\alpha}^{a}\right)=$ $i\left(x_{\beta}^{a}\right)$ holds for some $a \in C$, then it holds for all $a \in C$.

Applying the Erdös-Rado theorem to the sets $S_{a}=\left\{i\left(x_{\alpha}^{a}\right) \mid \alpha<\mathfrak{m}_{0}\right\}$ for $a \in C$, we obtain a subset $C^{\prime} \subseteq C$ with $\left|C^{\prime}\right|=\mathfrak{n}$ such that $\left(S_{a} \mid a \in\right.$ $C^{\prime}$ ) is a $\Delta$-system with kernel $D$. Again as in Theorem 1 , we can assume that if $i\left(x_{\alpha}^{a}\right) \in D\left(a \in C^{\prime}, \alpha<\mathfrak{m}_{0}\right)$, then $i\left(x_{\alpha}^{a}\right)=i\left(x_{\alpha}^{b}\right)$ for all $b \in C^{\prime}$. We will consider only the case that $I-D \neq \varnothing$. Choose $k \in I-D$, set $J=D \cup\{k\}$, and let $K$ be a $V$-free m-product of $\left(L_{i} \mid i \in J\right)$. Further, choose $e \in L_{k}$. Let $\varphi: L \rightarrow K$ be the m-homomorphism that maps $L_{i}$ identically if $i \in D$, and maps everything in $L_{i}$ to $e$ if $i \in$ $I-D$. If $a<b$ in $C^{\prime}$, then Lemma 1 guarantees that $\varphi(a) \neq \varphi(b)$. Therefore, $\left\{\varphi(a) \mid a \in C^{\prime}\right\}$ is a chain of cardinality $\mathfrak{n}$ in $K$, completing the proof.

Note that Corollary 1 of Theorem 1 also follows from Theorem 2 .

5. Proofs of Theorems 3 and 4. In order to develop a proof of Theorem 3, we will generalize the concepts and results in $\S 5$ of G. Grätzer and H. Lakser [8]. Let $\left(P_{i} \mid i \in I\right)$ be a family of posets with 0 and 1 . Let $k=0$ or 1 . For each $x$ in the direct product $\Pi\left(P_{i} \mid i \in I\right), s p_{k}(x)=\left\{i \in I \mid x_{i} \neq k\right\}$. Also, $\Pi_{\mathrm{m}}^{k}\left(P_{i} \mid i \in I\right)$ is the set of all $x \in \Pi\left(P_{i} \mid i \in I\right)$ for which $\left|s p_{k}(x)\right|<\mathfrak{m}$. The $\mathfrak{m}$-weak direct product of $\left(P_{i} \mid i \in I\right)$ is defined as 


$$
\Pi_{\mathrm{m}}\left(P_{i} \mid i \in I\right)=\Pi_{\mathrm{m}}^{0}\left(P_{i} \mid i \in I\right) \cup \Pi_{\mathrm{m}}^{1}\left(P_{i} \mid i \in I\right) .
$$

LEMMA 2. Let $\mathfrak{n}$ be a strongly $\mathfrak{m}$-inaccessible cardinal whose cofinality is greater than $2^{\mathrm{m}}$. If $\left(P_{i} \mid i \in I\right)$ is a family of posets with 0 and 1 satisfying the $\mathrm{n}$-chain condition, then $\Pi_{\mathrm{m}}\left(P_{i} \mid i \in I\right)$ satisfies the $\mathfrak{n}$-chain condition.

Proof. Suppose $C$ is a chain in $\Pi_{\mathrm{m}}\left(P_{i} \mid i \in I\right)$ of cardinality $\mathfrak{n}$, where each $P_{i}$ satisfies the $\mathfrak{n}$-chain condition. There is no loss in generality in assuming that $C \leqq \Pi_{\mathrm{m}}^{0}\left(P_{i} \mid i \in I\right)$. For $x \in C$, the sets $s p_{0}(x)$ each have cardinality less than $\mathfrak{m}$ and form a chain under inclusion; therefore, by the Erdös-Rado theorem (a proof without appeal to this theorem is not difficult), $\left|\left\{s p_{0}(x) \mid x \in C\right\}\right| \leqq 2^{\mathrm{m}}$. Thus, there is a chain $C^{\prime} \leqq C$ of cardinality $\mathfrak{n}$ and a set $J \subseteq I$ of cardinality $\mathfrak{m}_{0}<\mathfrak{m}$ such that $s p_{0}(x)=J$ whenever $x \in C^{\prime}$. For $i \in J$, let $C_{i}=\pi_{i}\left(C^{\prime}\right)$, where $\pi_{i}: \Pi\left(P_{i} \mid i \in I\right) \rightarrow P_{i}$ is the projection map; since each $C_{i}$ is a chain in $P_{i},\left|C_{i}\right|<\mathfrak{H}$. Choose $\mathfrak{n}_{0}<\mathfrak{n}$ such that $|C| \leqq \mathfrak{n}_{0}$ whenever $i \in J$. Since $C^{\prime}$ can be embedded in $\Pi\left(C_{i} \mid i \in J\right)$, we obtain $\left|C^{\prime}\right| \leqq \mathfrak{n}_{0}^{\mathfrak{m}_{0}}<\mathfrak{H}$. With this contradiction, the proof is complete.

Lemma 3. Let $\mathfrak{n}$ be a strongly $\mathfrak{m}$-inaccessible cardinal whose cofinality is greater than $2^{\mathrm{m}}$. There is a Boolean m-algebra in $\boldsymbol{D}_{\mathrm{m}}$ that satisfies the n-chain condition but contains a chain of cardinality $\mathfrak{n}^{\prime}$ for every $\mathfrak{n}^{\prime}<\mathfrak{n}$.

Proof. Any successor ordinal, considered as a (complete) chain, is isomorphic to an $\mathfrak{m}$-sublattice of a power set. For each $a<\mathfrak{n}$, let $B_{\mathrm{a}}$ be a Boolean $\mathfrak{m}$-algebra in $\boldsymbol{D}_{\mathrm{m}}$ that is $\mathrm{m}$-generated inside a Boolean mi-algebra $A$ in $D_{\mathrm{m}}$ by $C \cup\{0,1\} \cup\left\{c^{\prime} \mid c \in C\right\}$, where $C$ is a successor ordinal of cardinality $a$ and $c^{\prime}$ denotes the complement of $c$ in $A$. An $\mathfrak{m}$-polynomial in which $\mathfrak{m}_{0}<\mathfrak{m}$ variables appear can represent at most $\mathfrak{a}^{\mathrm{m}_{0}}$ elements of $B_{a}$. Since $\mathrm{a}^{\mathrm{m}_{0}}<\mathfrak{n}$ and there are $2^{\mathfrak{m}} \mathfrak{m}$-polynomials, it follows that $\left|B_{\mathrm{a}}\right|<\mathfrak{n}$. Then $B=\Pi_{\mathrm{m}}\left(B_{\mathrm{a}} \mid \mathfrak{a}<\mathfrak{n}\right)$ is a Boolean m-algebra in $D_{\mathrm{m}}$ and, by Lemma $2, B$ satisfies the $\mathfrak{n}$ chain condition.

Now we prove Theorem 3 . Let $B_{1}$ be a Boolean m-algebra in $\boldsymbol{D}_{\mathrm{m}}$ satisfying the condition of Lemma 3 . If $\boldsymbol{\aleph}_{\alpha}$ is the cofinality of $\mathfrak{n}$, we can write $\mathfrak{n}=\sum\left(\mathfrak{n}_{\beta} \mid \beta<\omega_{\alpha}\right)$, where $\mathfrak{n}_{\beta}<\mathfrak{n}$ for all $\beta<\omega_{\alpha}$. For each $\beta<\omega_{\alpha}$, let $C_{\beta} \subseteq B_{1}$ be a chain of cardinality $\mathfrak{n}_{\beta}$. Let $B_{2}$ be a Boolean m-algebra that is Boolean m-generated by the ordinal $\omega_{\alpha}+1$ inside a power set; then $\left|B_{2}\right|<\mathfrak{\pi}$. Further, let $L$ be the $V$-free m-product of $B_{1}$ and $B_{2}$. For $\beta<\omega_{\alpha}$, let $C_{\beta}^{\prime}=\{(x \vee \beta) \wedge$ $\left.(\beta+1) \mid x \in C_{\beta}\right\}$; then $C=\bigcup\left(C_{\beta}^{\prime} \mid \beta<\omega_{\alpha}\right)$ is a chain in $L$. Let $\psi: B_{2} \rightarrow \mathbf{2}$ 
be an m-homomorphism such that $\psi(\beta)=0$ and $\psi(\beta+1)=1$. We now define the m-homomorphism $\varphi: L \rightarrow B_{1} \cup\{0,1\}$ by $\varphi(x)$ if $x \in B_{1}$, and $\varphi(x)=\psi(x)$ if $x \in B_{2}$. Since $\varphi((x \vee \beta) \wedge(\beta+1))=x$, it now follows that $\left|C_{\beta}^{\prime}\right|=\mathfrak{n}_{\beta}$. Therefore, $|C|=\mathfrak{n}$, completing the proof.

Theorem 4 is easier to prove. Indeed, if $\mathfrak{n} \leqq \mathfrak{m}$, the $V$-free $\mathfrak{m}$-lattice with $\mathfrak{n}$ generators $\left\{x_{\alpha} \mid \alpha<\mathfrak{n}\right\}$ contains the chain $\left\{y_{\alpha} \mid \alpha<\mathfrak{n}\right\}$ of cardinality $\mathfrak{n}$, where $y_{\alpha}=\mathrm{V}\left(x_{\beta} \mid \beta \leqq \alpha\right)$ whenever $\alpha<\mathfrak{n}$. If $\mathfrak{n}>\mathfrak{m}$, then $\mathfrak{n}=\Sigma\left(\mathfrak{n}_{\alpha} \mid \alpha<\mathfrak{m}_{0}\right)$, where $\mathfrak{n}_{\alpha}<\mathfrak{n}$ for all $\alpha<\mathfrak{m}_{0}$. Let $C$ and $C_{\alpha}$ be successor ordinals of cardinality $\mathfrak{m}_{0}$ and $\mathfrak{n}_{\alpha}$, respectively, where $\alpha<\mathfrak{m}_{0}$. The proof is completed similarly as in Theorem 3 by showing that each $C_{\alpha}$ can be embedded into the interval $(\alpha, \alpha+1)$ in the $V$-free m-product of $C$ and the $C_{\alpha}\left(\alpha<\mathfrak{m}_{0}\right)$.

\section{Open problems.}

Problem 1. Is every $V$-free m-lattice a union of $2^{\mathrm{m}}$ antichains? First we show that this holds for $\mathfrak{m}=\aleph_{0}$.

Proposition 1. Any V-free lattice is a countable union of antichains.

Proof. Let $L$ be the $V$-free lattice generated by a set $X$. Let $p$ be a polynomial in variables $x_{1}, x_{2}, \cdots, x_{n}$ and let $S$ be the set of all $a \in L$ that have a proper representation of the form $a=p\left(x_{1}, \cdots\right.$, $x_{n}$ ) where $x_{i} \in X, 1 \leqq i \leqq n$. It is enough to show that $S$ is an antichain. Let $\sigma$ be a permutation of $\{1,2, \cdots, n\}$. For $a=\boldsymbol{p}\left(x_{1}, \cdots\right.$, $\left.x_{n}\right)$, we write $\sigma a$ for $p\left(x_{\sigma(1)}, \cdots, x_{\sigma(n)}\right)$. If $a \leqq \sigma a$, then $\sigma a \leqq \sigma^{2} a, \cdots$, $\sigma^{n-1} a \leqq \sigma^{n} a=a$, from which it follows that $a=\sigma a$. (F. Galvin and B. Jónsson used similar reasoning in [4].) Now, let $a=\boldsymbol{p}\left(x_{1}, \cdots\right.$, $\left.x_{n}\right)$ and $b=\boldsymbol{p}\left(y_{1}, \cdots, y_{n}\right)$ be proper representations with $x_{i}, y_{i} \in X$, $1 \leqq i \leqq n$, and suppose that $a \leqq b$. Let $A=\left\{x_{1}, \cdots, x_{n}\right\}$ and $B=$ $\left\{y_{1}, \cdots, y_{n}\right\}$. We can assume there is an integer $k$ with $0 \leqq k \leqq n$ and there are elements $z_{1}, \cdots, z_{k} \in X$ such that $A-B=\left\{z_{1}, \cdots, z_{k}\right\}$ and $A \cap B=\left\{y_{k+1}, \cdots, y_{n}\right\}$. Applying the obvious endomorphism of $L$ to the inequality $a \leqq b$, we obtain $\boldsymbol{p}\left(x_{1}, \cdots, x_{n}\right) \leqq \boldsymbol{p}\left(z_{1}, \cdots, z_{l}\right.$, $\left.y_{k+1}, \cdots, y_{n}\right)$; by the previous case, $a=p\left(z_{1}, \cdots, z_{k}, y_{k+1}, \cdots, y_{n}\right)$. Let $\phi$ be the endomorphism of $L$ that maps $z_{i}$ to $y_{i}$, and vice-versa $(1 \leqq i \leqq k)$, and maps all other elements of $X$ identically. Applying $\varphi$ to the inequality $\boldsymbol{p}\left(z_{1}, \cdots, z_{k}, y_{k+1}, \cdots, y_{n}\right) \leqq \boldsymbol{p}\left(y_{1}, \cdots, y_{n}\right)$, we obtain $b \leqq a$, completing the proof.

The following example shows that similar reasoning will not settle the uncountable case. (For notational simplicity, we only deal with the $\mathfrak{m}=\aleph_{1}$ case.) 
Let $V$ be a nontrivial variety of $\aleph_{1}$-lattices and let $L$ be a $V$ free lattice generated by an infinite set $X$. We show that, in contrast with the $\mathfrak{m}=\boldsymbol{\aleph}_{0}$ case, permutations of $X$ can create distinct comparable elements in $L$. Let $\boldsymbol{p}$ and $\boldsymbol{q}$ be $\boldsymbol{\aleph}_{1}$-polynomials in variables $\left\{\boldsymbol{x}_{n} \mid n<\omega\right\}$ such that $\boldsymbol{p} \leqq q$ holds in $\boldsymbol{V}$ (for any substitution) but $\boldsymbol{p}=\boldsymbol{q}$ does not (for example, $\boldsymbol{x}_{0}$ and $\boldsymbol{x}_{0} \vee \boldsymbol{x}_{1}$ ). Let $x_{n}^{i}$ be distinct elements of $X$ for $i \in Z$ (the integers) and $n<\omega$. Further, let $p_{i}=\boldsymbol{p}\left(x_{n}^{i} \mid n<\omega\right)$ and $q_{i}=q\left(x_{n}^{i} \mid n<\omega\right)$. If

$$
a=\bigvee\left(p_{i} \mid i \leqq 0\right) \vee \bigvee\left(q_{i} \mid i>0\right)
$$

and

$$
b=\bigvee\left(p_{i} \mid i<0\right) \vee \bigvee\left(q_{i} \mid i \geqq 0\right),
$$

then $a \leqq b$ and $b$ can be obtained from $a$ by suitably permuting the elements of $X$. If $a=b$, we obtain $p_{0}=q_{0}$ by mapping each $x_{n}^{i}$ $(i \neq 0, n<\omega)$ to $\wedge\left(x_{n}^{0} \mid n<\omega\right)$. This would mean that $\boldsymbol{p}=\boldsymbol{q}$ holds in $V$, contrary to assumption. Therefore, $a<b$. In fact, a chain isomorphic to the reals $R$ can be obtained from $a$ by suitable permutations of $X$. (Let $f: Z \rightarrow Q$ be a bijection, and for $y \in R$, let $a_{y}=\mathrm{V}\left(r_{i} \mid i \in Z\right)$, where $r_{i}=p_{i}$ if $f(i)<y$ and $r_{i}=q_{i}$ otherwise.)

Problem 2. Let $\mathfrak{n}$ be regular and $>\mathfrak{m}$. Do $V$-free m-products preserve the $\mathfrak{n}$-chain condition?

This problem was answered affirmatively for $\mathfrak{m}=\boldsymbol{\aleph}_{0}$ and $\boldsymbol{V}=\boldsymbol{D}$ by G. Grätzer and H. Lakser [6]. For $\mathfrak{m}=\boldsymbol{S}_{0}$ and $\boldsymbol{V}=\boldsymbol{L}$, an affirmative answer was found by M. E. Adams and D. Kelly [1] by separately proving the following two statements:

(i) The free product of a family $\left(L_{i} \mid i \in I\right)$ of lattices is isomorphic to a subposet of the completely free lattice generated by the poset $\dot{U}\left(L_{i} \mid i \in I\right)$.

(ii) If a poset $X$ satisfies the $\mathfrak{n}$ chain condition, then so does the completely $V$-free lattice generated by $X$.

It is shown in [6] that the statement corresponding to (i) for m-lattices is valid. On the other hand, the following example shows that the analogue of (ii) is false.

Let $\mathfrak{m}$ and $\mathfrak{n}$ be uncountable cardinals and consider the poset $X=\left\{x_{n}^{\alpha} \mid n<\omega, \alpha<\mathfrak{n}\right\}$ where $x_{\mathfrak{m}}^{\alpha}<x_{\mathfrak{m}}^{\beta}$ iff $\mathfrak{m}<\mathfrak{n}$ and $\alpha<\beta$. Then $X$ contains only countable chains but the completely $V$-free lattice $L$ generated by $X$ contains a chain of cardinality $\mathfrak{n}$, where $V$ is an arbitrary nontrivial variety of m-lattices. For $\alpha<\mathfrak{n}$ let $y_{\alpha}=$ $\mathrm{V}\left(x_{n}^{\alpha} \mid n<\omega\right)$; clearly, $\left\{y_{\alpha} \mid \alpha<\mathfrak{n}\right\}$ is a chain in $L$. Let $\alpha<\beta<\mathfrak{n}$. The isotone map $\varphi: X \rightarrow \mathbf{2}$ defined by $\varphi\left(x_{n}^{\gamma}\right)=0$ if $\gamma \leqq \alpha$ and $\varphi(x)=1$ 
for $x \in X$ otherwise extends to an $\mathfrak{m}$-homomorphism of $L$ onto 2 that maps $y_{\alpha}$ to 0 and $y_{\beta}$ to 1 ; thus, $y_{\alpha} \neq y_{\beta}$.

Problem 3. Is every $\mathrm{m}$-complete chain contained in a Boolean $\mathfrak{m}$-algebra in $D_{\mathrm{m}}$ ?

If $\mathfrak{m}=\mathfrak{n}^{+}$, a Boolean $\mathfrak{m}$-algebra in $\boldsymbol{D}_{\mathfrak{m}}$ is called $\mathfrak{n}$-representable by R. Sikorski [10]. If, for any two distinct elements of an $\mathfrak{m}$ lattice $L$, there is an m-homomorphism from $L$ onto 2 separating the two elements, then $L$ is in $\boldsymbol{D}_{\mathrm{m}}$. Thus, as observed in the proof of Lemma 3, any successor ordinal is an $\mathfrak{m}$-sublattice of a power set. It also follows that $\boldsymbol{D}_{\mathrm{u}}$ contains every m-complete chain. (Replace each element of an m-complete chain $C$ by two elements, forming the chain $C^{\prime}$; then $C^{\prime}$ is an m-sublattice of a power set and the obvious map from $C^{\prime}$ to $C$ is an m-homomorphism.) Since the embedding of a chain into the Boolean algebra that it $R$-generates preserves all existing joins and meets (see [5]), any m-complete chain is an m-sublattice of a Boolean m-algebra. However, the following example shows that m-congruences of maximal chains need not extend to $\mathfrak{m}$-congruences of Boolean $\mathfrak{m}$-algebras. (Contrast with the $\mathfrak{m}=\aleph_{0}$ case in [5].) Let $B$ be the power set of $[0,1]$ and let $C$ be the maximal chain in $B$ consisting of all intervals of the form $[0, x)$ or $[0, x]$, where $x \in[0,1]$. The $\mathfrak{m}$-homomorphism that only collapses $[0, x)$ and $[0, x], 0 \leqq x \leqq 1$, maps $C$ onto $[0,1]$. Yet, if $\mathfrak{m} \geqq\left(2^{\aleph_{0}}\right)^{+}$, any $\mathfrak{m}$-congruence of $B$ that collapses $[0, x)$ and $[0, x]$, $0 \leqq x \leqq 1$, collapses all of $B$ since $[0,1] \leqq \bigcup([0, x]-[0, x) \mid 0 \leqq x \leqq 1$.)

\section{REFERENCES}

1. M. E. Adams and D. Kelly, Chain conditions in free products of lattices, Algebra Universalis, 7 (1977), 235-243.

2. W. W. Confort and S. Negrepontis, The Theory of Ultrafilters, Springer-Verlag, New York, 1974.

3. P. Erdös and R. Rado, Intersection theorems for systems of sets II, J. London Math. Soc., 44 (1969), 467-479.

4. F. Galvin and B. Jónsson, Distributive sublattices of a free lattice, Canad. J. Math., 13 (1961), 265-272.

5. G. Grätzer, General Lattice Theory, Birkhäuser Verlag, Basel, 1979.

6. G. Grätzer and D. Kelly, Free m-product of lattices, Colloq. Math., to appear.

7. G. Grätzer and D. Kelly, A normal form theorem for lattices completely generated by a subset, Proc. Amer. Math. Soc., 67 (1977), 215-218.

8. G. Grätzer and $\mathrm{H}$. Lakser, Chain conditions in the distributive free product of lattices, Trans. Amer. Math. Soc., 144 (1969), 301-312.

9. B. Jónsson, Relatively free products of lattices, Algebra Universalis, 1 (1972), 362-373.

10. R. Sikorski, Boolean Algebras, 3rd edition, Springer-Verlag, New York, 1969.

Received March 21, 1978 and in revised form December 29, 1978. The research of the authors was supported by the National Research Council of Canada. 



\section{PACIFIC JOURNAL OF MATHEMATICS}

EDITORS

DONALD BABBITT (Managing Editor)

University of California

Los Angeles, California 90024

Hugo RossI

University of Utah

Salt Lake City, UT 84112

C. C. MOORE and ANDREW OGG

University of California

Berkeley, CA 94720

\section{J. DUGUNDJI}

Department of Mathematics University of Southern California Los Angeles, California 90007

R. Finn and J. Milgram Stanford University

Stanford, California 94305

\section{ASSOCIATE EDITORS}
E. F. BECKENBACH
B. H. Neumann
F. WOLF
K. YoSHIDA

\section{SUPPORTING INSTITUTIONS}

UNIVERSITY OF BRITISH COLUMBIA

CALIFORNIA INSTITUTE OF TECHNOLOGY

UNIVERSITY OF CALIFORNIA

MONTANA STATE UNIVERSITY

UNIVERSITY OF NEVADA, RENO

NEW MEXICO STATE UNIVERSITY

OREGON STATE UNIVERSITY

UNIVERSITY OF OREGON
UNIVERSITY OF SOUTHERN CALIFORNIA

STANFORD UNIVERSITY

UNIVERSITY OF HAWAII

UNIVERSITY OF TOKYO

UNIVERSITY OF UTAH

WASHINGTON STATE UNIVERSITY

UNIVERSITY OF WASHINGTON 


\section{Pacific Journal of Mathematics}

\section{Vol. 83, No. 1 \\ March, 1979}

Richard Neal Ball, Topological lattice-ordered groups ............... 1

Stephen Berman, On the low-dimensional cohomology of some

infinite-dimensional simple Lie algebras .................. 27

R. P. Boas and Gerald Thomas Cargo, Level sets of derivatives ......... 37

James K. Deveney and John Nelson Mordeson, Splitting and modularly

perfect fields......................................

Robert Hugh Gilman and Ronald Mark Solomon, Finite groups with small

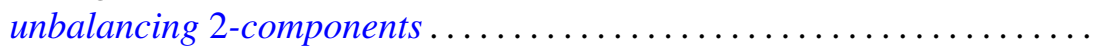

George Grätzer, Andras Hajnal and David C. Kelly, Chain conditions in free products of lattices with infinitary operations..................

Benjamin Rigler Halpern, Periodic points on tori ..................

Dean G. Hoffman and David Anthony Klarner, Sets of integers closed under

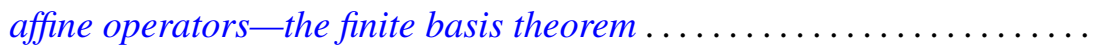

Rudolf-Eberhard Hoffmann, On the sobrification remainder ${ }^{s} X-X \ldots \ldots$

Gerald William Johnson and David Lee Skoug, Scale-invariant

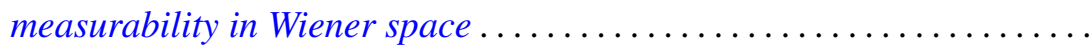

Michael Keisler, Integral representation for elements of the dual of

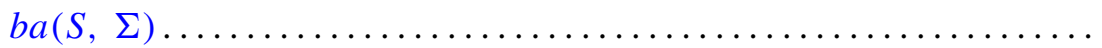

Wayne C. Bell and Michael Keisler, A characterization of the representable Lebesgue decomposition projections ................

Wadi Mahfoud, Comparison theorems for delay differential equations ...

R. Daniel Mauldin, The set of continuous nowhere differentiable functions .

Robert Wilmer Miller and Mark Lawrence Teply, The descending chain condition relative to a torsion theory...

Yoshiomi Nakagami and Colin Eric Sutherland, Takesaki's duality for regular extensions of von Neumann algebras ........ .

William Otis Nowell, Tubular neighborhoods of Hilbert cube manifolds ...

Mohan S. Putcha, Generalization of Lentin's theory of principal solutions of word equations in free semigroups to free product of copies of positive reals under addition

Amitai Regev, A primeness property for central polynomials . ...

Saburou Saitoh, The Rudin kernels on an arbitrary domain. . .

Heinrich Steinlein, Some abstract generalizations of the

Ljusternik-Schnirelmann-Borsuk covering theorem . . . 